\title{
Design of Seismic Intensity Rapid Report Platform
}

\author{
Guo Yunkai ${ }^{1, \star}$, Tan Qiao $^{1}$, Li Zhitao $^{1}, \mathrm{Fu} \mathrm{Jihua}^{1}$, Xiao Ke $^{2}$ \\ ${ }^{1}$ Institute of Crustal Dynamics, China Earthquake Administration, Beijing, 100085, China \\ ${ }^{2}$ North China University of Technology, Beijing, 100041, China \\ email: top_usky@126.com
}

Keywords: Earthquake, Rapid Report Platform, Seismic Intensity, Intensity Sensor

\begin{abstract}
Seismic intensity rapid report is one of the essential parts of the earthquake disaster relief and plays a baton role. In terms of serving the needs for earthquake early warning, emergency response, and seismic mobile observation, seismic network observation, a new seismic intensity rapid report platform is developed for the seismic intensity sensor network. The platform has a complete seismic intensity monitor system, and can achieve accurate and efficient intensity data recovery and analysis, built rapid and efficient intensity report system. Some key functions were utilized to integrate the platform, such as data collection, real-time data analysis, graphic display and intensity rapid reporting. A serial of experiments were carried out and the results showed that the platform could fulfill the purpose of seismic emergency response and deserve to be widely popularized.
\end{abstract}

\section{Introduction}

China is one of the earthquake-prone countries in the world, whose earthquake has wide distribution, high frequency, high intensity, heavy disaster wait for characteristics. Strengthening seismic monitoring, earthquake early warning and disaster prevention are the inevitable requirements of social economic development ${ }^{[1,2]}$.

In recent decades, along with the improvement of the global economy as well as deep understanding of the earthquake monitoring, great importance has been attached to the construction of the world's earthquake monitor network. Meanwhile, with the development of digital seismic intensity sensors, digital communication technology and computer software and hardware, the purpose of earthquake monitor network is no longer confined to provide experimental data for the study of earthquake engineering, but extends to the service for earthquake prevention and disaster reduction. Using seismic intensity rapid report based the real-time or near real-time ground motion record to provide first-hand information for earthquake emergency is a concrete embodiment.

Therefore, development of the seismic rapid report system, which is composed by seismic intensity sensors distributed in different locations and a seismic intensity rapid report platform managing the sensors, is an important business of earthquake prevention and disaster reduction. The earthquake rapid report platform is a management system for the seismic intensity sensor network. It transfers and collects the seismic record data from the high-performance intensity sensors via network, and offers further data analysis and release function.

Based on the practical requirements of building comprehensive seismic intensity rapid report system, this paper describes the design and functions of the seismic intensity rapid report platform in detail. The rest parts of this paper are organized as follows: section II introduces the hardware system of seismic intensity sensor and its workflow; The seismic intensity rapid report platform is described in structure ,function, as well as principle and feature of design in the section III; then section IV carries out testing experiments combined with the intensity sensors laid Guangyuan city in northern Sichuan province, China; Finally, section V draws the conclusions.

\section{Seismic Intensity Sensor}

Design of Hardware System. As shown in the figure 1, the hardware system of the seismic intensity sensor is composed of the ARM9 processor, the acceleration sensor, the data acquisition 
module(AD), the mobile communication module, IPv4/IPv6 network module, ZigBee module, GPS module and power management module ${ }^{[3,4,5]}$.

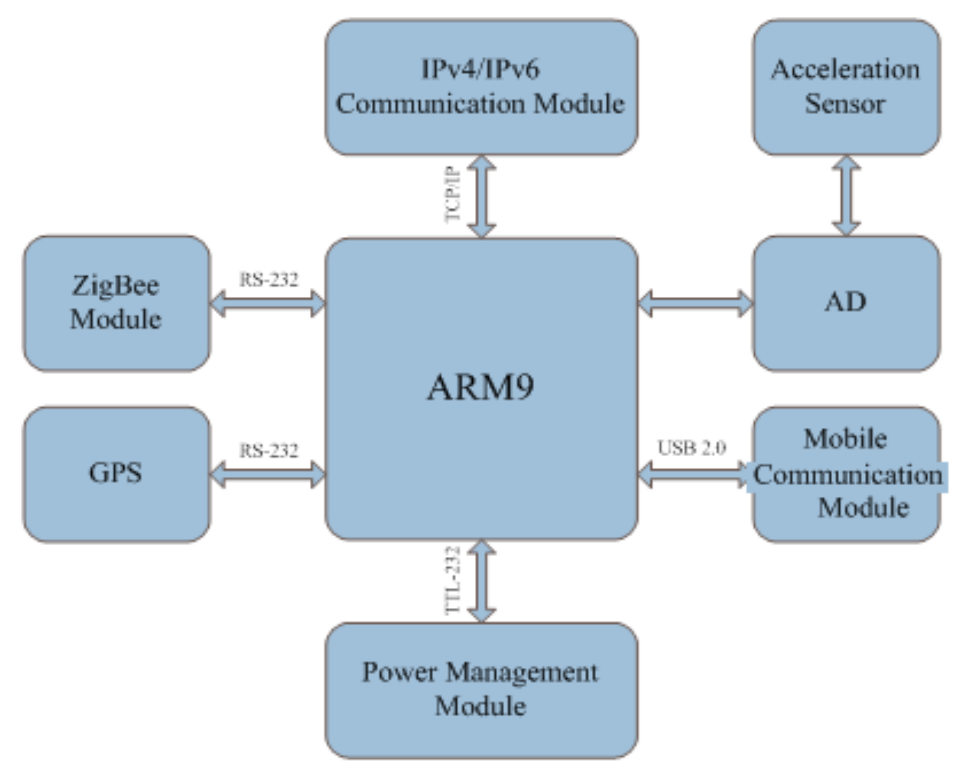

Figure 1: Overall structure of seismic intensity sensor

The ARM9 module is a low-power processor and integrates NAND Flash storage medium and NOR Flash. The acceleration sensor can measure the ground motion in the $\mathrm{X}, \mathrm{Y}, \mathrm{Z}$ directions to provide data for the processor. The processor calls corresponding algorithms to convert the data into intensity values. The data acquisition module uses the 24 bit precision $\mathrm{AD}$ and can dispel the signal distortion. The GPS module is utilized to obtain the sensors' location such as longitude and latitude. ZigBee is a short-range, low-power wireless communication technology. ZigBee module provides transfer functionality for the rapid report platform. IPv4 / IPv6 network module provides reliable data channel for uploading seismic disaster information.

Workflow of Seismic Intensity Sensor.

Workflow of seismic intensity sensor that is illustrated in figure 2 includes the following four stages.

(1).Signal maintenance stage

Intensity sensor sends signal every two minutes, and the server maintains the communication with the sensor via the heartbeat signal.

(2). State maintenance stage

Intensity sensor sends a state message every three hours. The server receives the state signal, then holds and updates the state information.

(3).Data reporting stage

Intensity sensor obtains the seismic data and sends the data to the server. The server requests the subsequent waveform data from the intensity sensor. Then the sensor will transmits waveform data divided into 75 frames.

(4).Data analysis stage

After receiving graphics data, the server enters the determination and analysis process via the background database. 


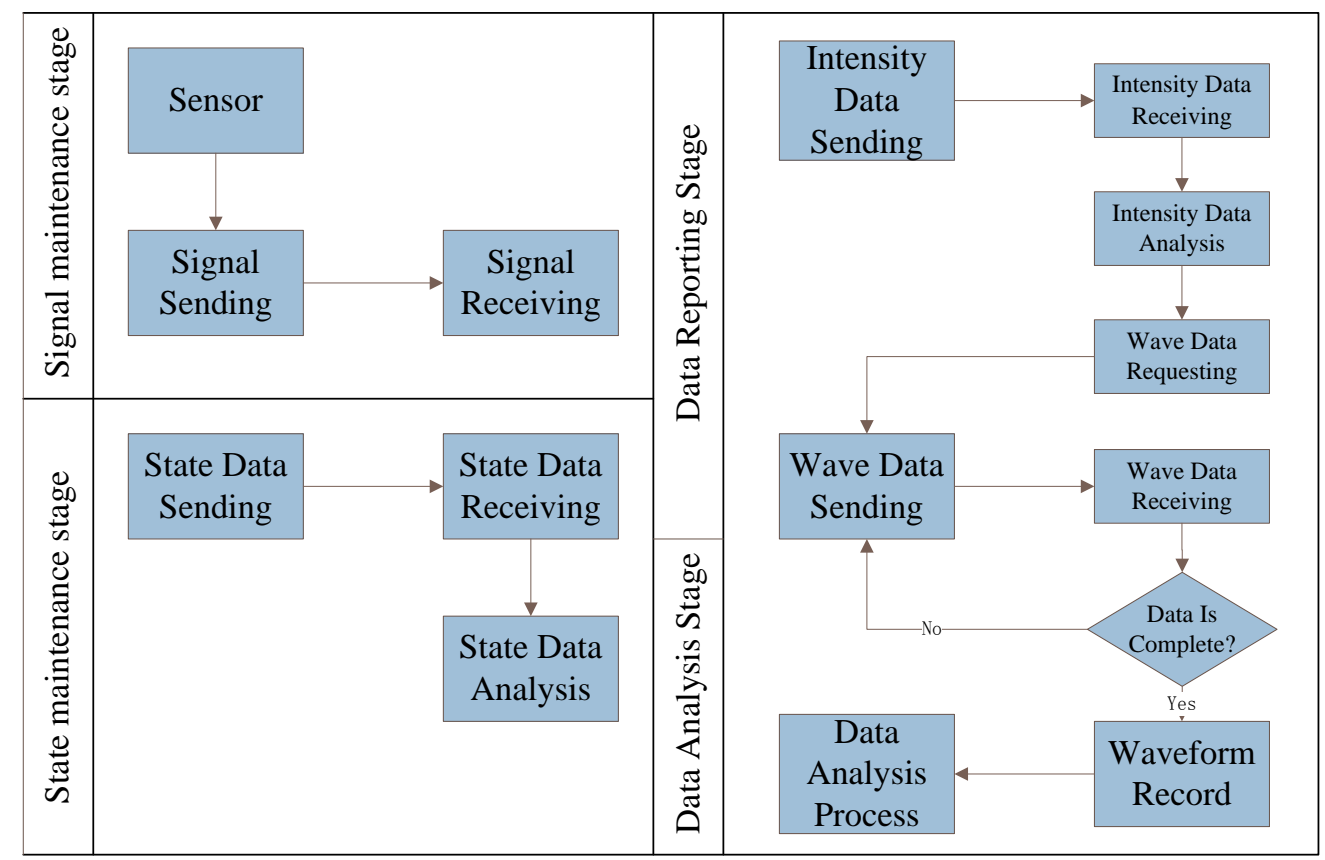

Figure 2: Workflow of seismic intensity Sensor

\section{The Seismic Intensity Rapid Report Platform}

The seismic intensity rapid report platform acts as an intermediate layer between the user and the sensor, providing an interactive platform for the sensor operation, seismic data acquisition, analysis and graphic display. The workflow diagram of the management platform for the seismic intensity rapid report is shown in figure 3 .

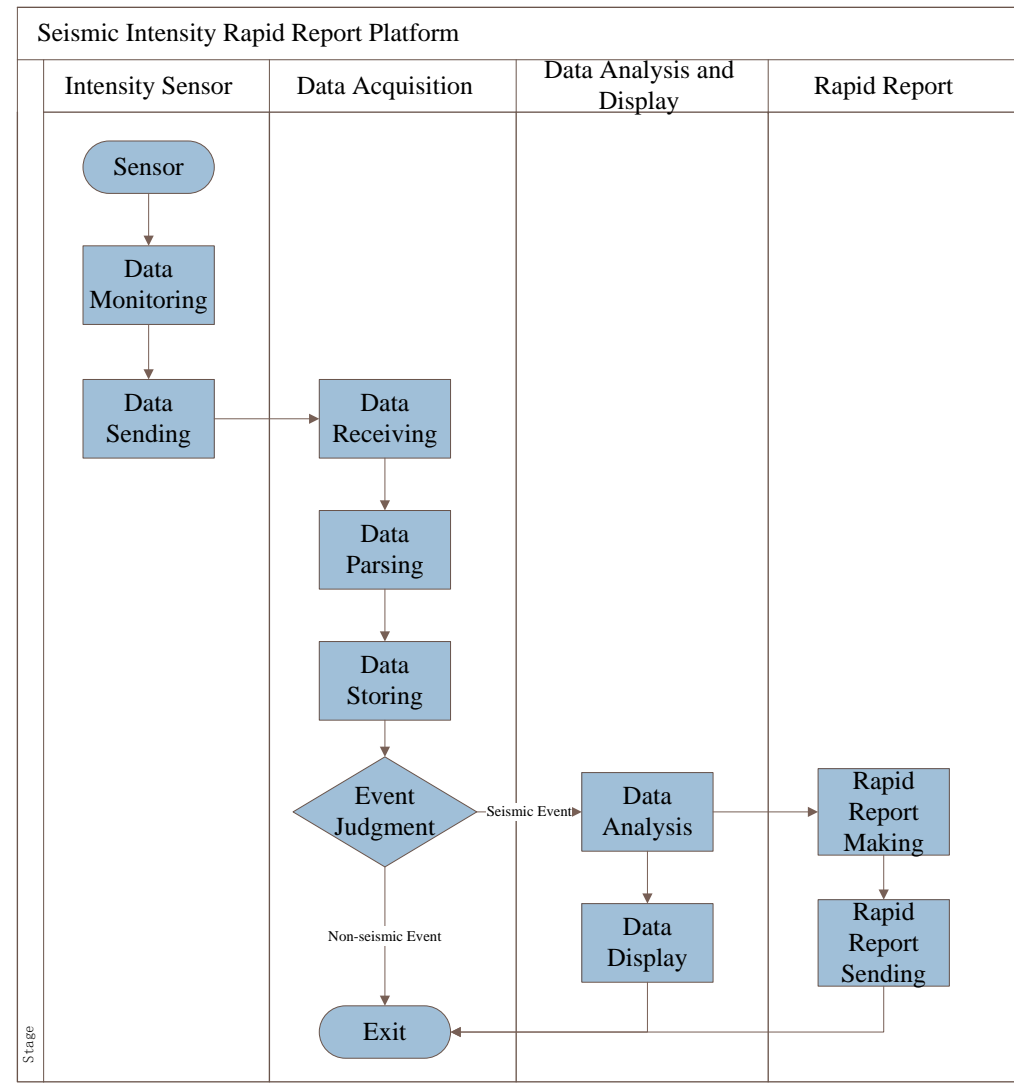

Figure 3: The workflow diagram of the management platform for seismic intensity rapid report 


\section{(1). Data Acquisition}

Data acquisition platform is a service module for data transmission with intensity sensor, mainly receiving, analyzing and storing the seismic data. First, data acquisition platform starts data receiving monitor. Then intensity sensor collects data during an earthquake and sends the data to the data acquisition platform. The platform processes the received data in the background eventually. In the process, this module will parse the received data, then re-organize data depending on a standard system format and output specification format data. After data acquisition platform successfully parsed the data in the standard format, it will write the specification format data to the storage system via calling the memory interface. The operation of this stage shall take the data receiving in high concurrency situation into account. It needs to ensure system stability and data integrity in this situation. In addition, high concurrency of data will bring about great pressure to the IO writing ability of the storage system. The caching mechanism or distributed data storage will be used if necessary.

\section{(2). Data Analysis}

The original data gathered from the sensor will be further analyzed and processed by the data analysis module according to some related algorithms and requirements. The data analysis module will obtain original data of a certain period from the storage system, and then call the related analysis algorithms to process the data. After data processing, the data will be stored in the storage system for graphic display and rapid report. In the process, the platform will analyze the data based on related algorithms and practical requirements to ensure the accuracy and reliability of the results.

\section{(3). Rapid Report}

After final data are analyzed, the rapid report management module will make rapid report file in a very short time scale. The rapid report module firstly obtains data from the storage system, and then calls the system rapid report template, fills the data into the template to form a rapid report file and send it to the manager. In the process, the transmit queue should be monitored and reported. In addition, transmit capability should be taken into account in the high concurrency situation.

\section{(4). Graphic Display}

The function of the module is to provide a graphic display of the parsed data for administrators and professional users analyzing the data situation, and produce important information that the professional users want. The major display information includes: sensor ID, latitude and longitude of the sensor, the intensity values generated in the $\mathrm{X}, \mathrm{Y}, \mathrm{Z}$ directions, and seismic intensity distribution contours. Seismic intensity distribution contours are based primarily on business data collected from the sensors. Users can obtain the distribution of the sensors and the corresponding size of the seismic intensity. In this process, it is needed to update the graphics in real-time, and when necessary to save the graphics for high concurrency access.

\section{Experiments}

In order to verify functions of the seismic intensity rapid report platform in detail, a series of testing experiments combined with the intensity sensors laid Guangyuan city in northern Sichuan province were carried out.

The intensity sensors obtained the signal of artificial ground motion in the different site and sent the original data to the seismic intensity rapid report platform via an IPv4/IPv6 network. Then the platform parsed and analyzed the data in a short time. After data was analyzed, rapid report files were made and sent to related receivers for warning. At the same time, the intensity values waveform diagram of the $\mathrm{X}, \mathrm{Y}, \mathrm{Z}$ directions and the seismic intensity distribution contours were displayed in the platform. The intensity values waveform diagram of the $\mathrm{X}, \mathrm{Y}, \mathrm{Z}$ directions(N32 $\left.23^{\prime} 60.00^{\prime \prime}, \mathrm{E} 105^{\circ} 47^{\prime} 60.00^{\prime \prime}\right)$ is shown in figure 4 and the seismic intensity distribution contours is shown in figure 5. 


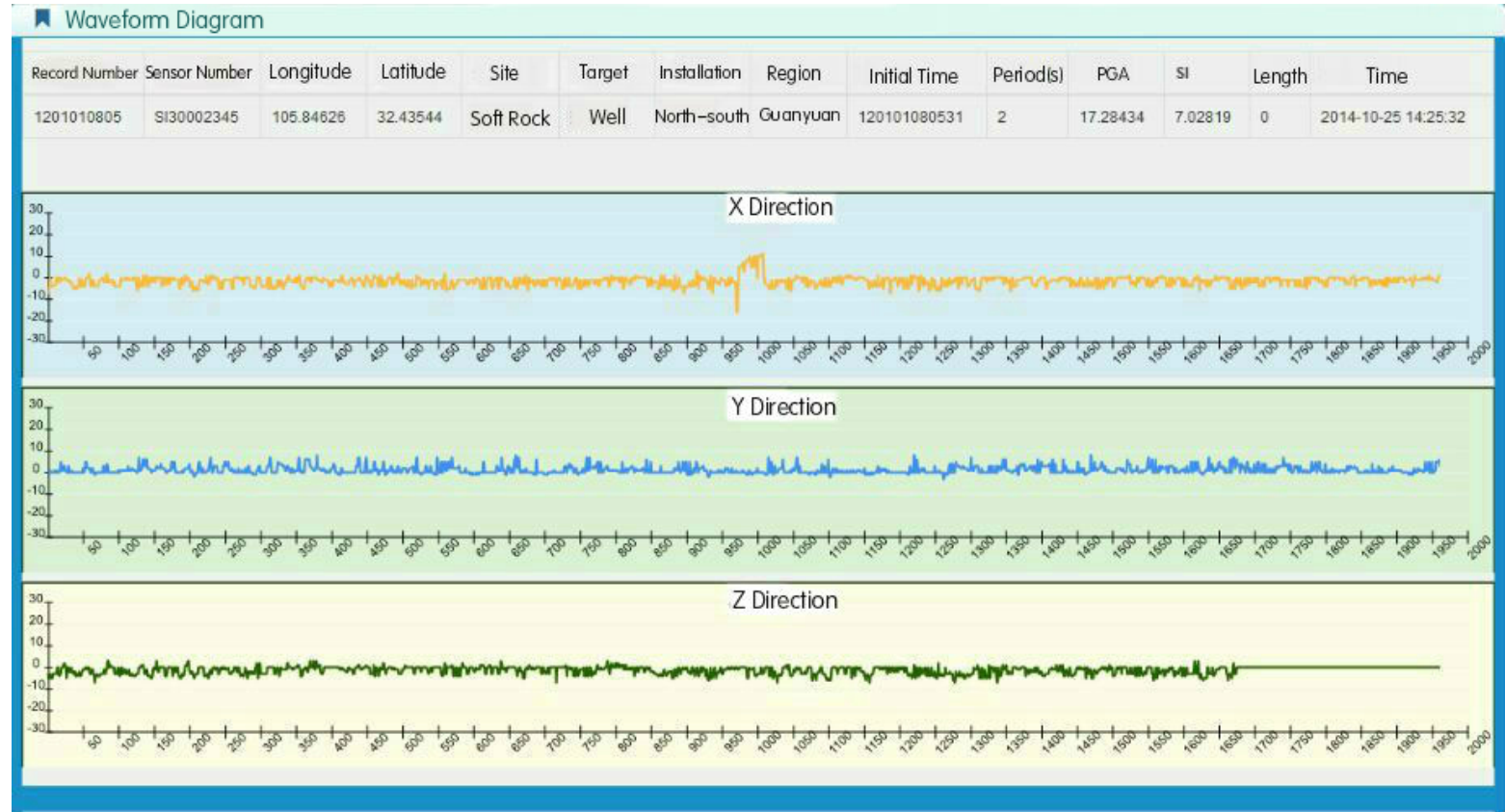

Figure 4: The intensity values waveform diagram of the $\mathrm{X}, \mathrm{Y}, \mathrm{Z}$ directions

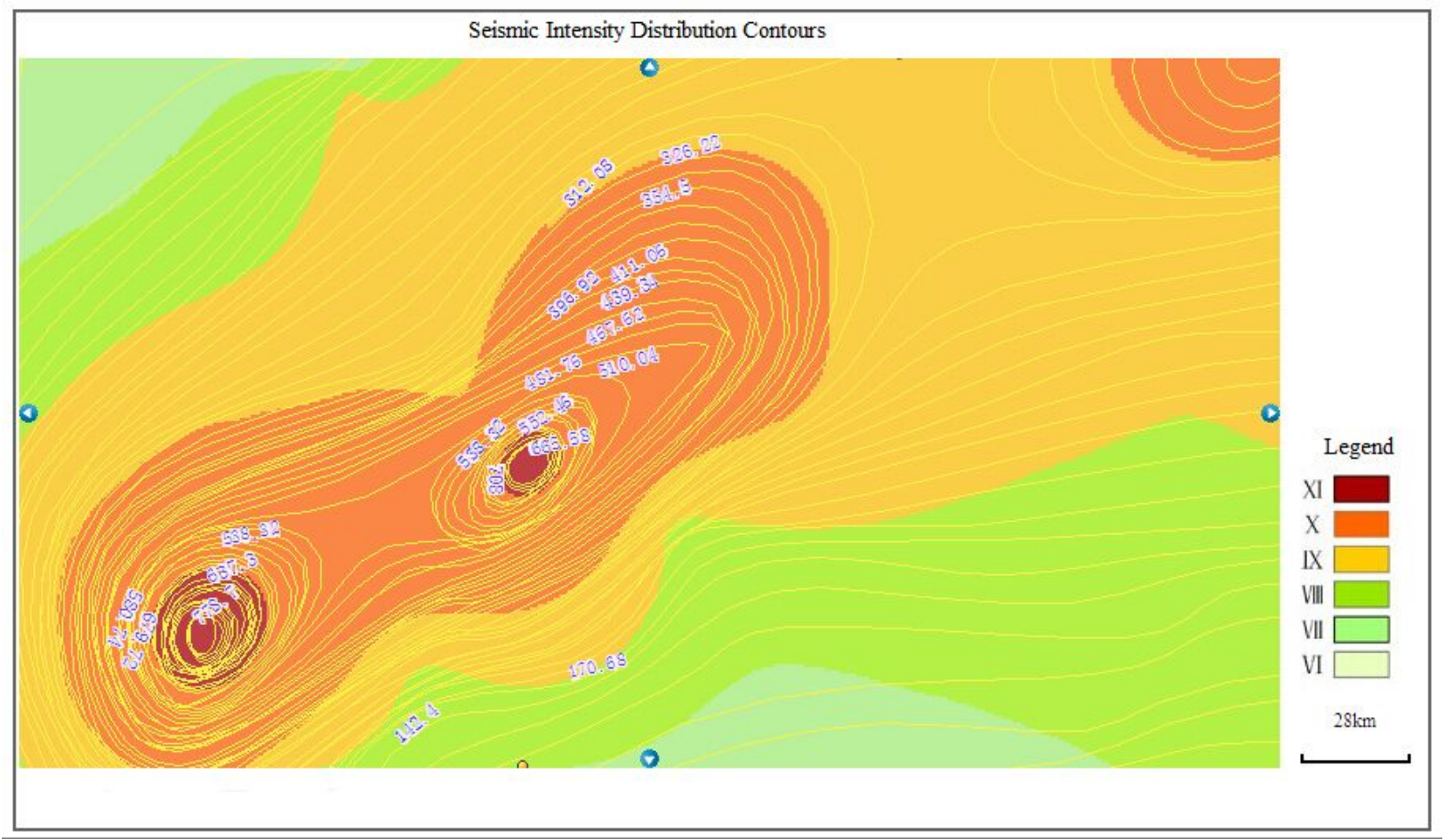

Figure 5: The seismic intensity distribution contours

\section{Conclusion}

The seismic intensity rapid report platform is a combination of various technologies such as the seismic monitoring instrumentation technology, sensor technology, embedded computing technology, distributed information processing technology, communication technology, network technology. The application of the advanced network in the seismic intensity rapid report platform achieves the real network of the earthquake monitoring system. In consequence, resources of seismic information are widely used in the construction of society and economy. The seismic intensity rapid report platform has the following characteristics:

1) Adaptability of network environment: Because the intensity sensors will be placed in the remote field, there are some limitation in the quality of the communication network. The platform 
has fully considered the data transmission delay and interrupt and so on to ensure the system stability and data integrity.

2) Efficiency of data analysis: On account of the disruptive affects of seismic disasters, data analysis of the platform with a certain accuracy an efficiency can provide an effective guarantee for the rapid processing of seismic disasters.

3) Specification of data transmission: The data sent by the platform matches various data standard, so it can adopt to the specification of various systems and platforms for sending and receiving data.

4) Remote management function: Remote monitor management technology is applied to managing the intensity sensors in the platform.

5) User friendly: With its favorable operability, the platform has better practical value.

\section{Acknowledgement}

This paper is supported by the research grant from Institute of Crustal Dynamics, China Earthquake Administration (No.ZDJ 2012-18).

\section{References}

[1] Tan Qiao,Wang Jian-jun,Liu Guan-zhong,Wu Rong-hui.Design of management system for the earthquake intensity sensor network based on IPv6[J].Transducer and Microsystem Technologies, 2010, 29(10):103-105.

[2] Lian Weiping,Liu Juhou.Application of IPv6 and NGN to the information network of earthquake preparedness and disaster mitigation[J].ACTA Seismologica Sinica, 2008, 21(6):665-669.

[3] Fu Jihua, Wang Jianjun, Li Zhitao, Liu Xiaoxi, Wu Ronghui. A new seismic emergency auto-handling instrument for the lifeline engineering: low cost embedded system solution[C]. International Conference on Intelligent Computation Technology and Automation, 2010, 1: 242 245. (EI)

[4] Fu Jihua, Wang Jianjun, Li Zhitao, Liu Xiaoxi, Wang Zhongyu. Intelligent seismic sensor with double three component MEMS accelerometers[C]. Proc. SPIE, 2010, 754422: 1 - 6. (EI)

[5] Wang Xi-zhen,Teng Yun-tian.New technology of seismic sensors and its development [J].Progress in geophysics, 2010, 25(2):478-485. 